\title{
A CLASS OF NONLINEAR ELLIPTIC PROBLEMS WITH NONCONVEX CONSTRAINTS AND APPLICATIONS
}

\author{
$b y$ N. CHEMETOV and J. F. RODRIGUES
}

(Received 31st July 1996)

\begin{abstract}
Conditions for the existence of solutions of a class of elliptic problems with nonconvex constraints are given in the general framework of pseudo-monotone operators. Applications are considered in unilateral problems of free boundary type, yielding the solvability of a Reynold's lubrication model and of a biological population problem with nonlocal terms and global constraints.
\end{abstract}

1991 Mathematics subject classification: 35A15, 35J85, 49M29, $35 \mathrm{R} 35$.

Variational problems with side conditions in the classical calculus of variations lead naturally to elliptic equations, as the Euler-Lagrange equations of the minimization problems, and to the associated Lagrange multipliers, as formal derivatives of the infimum with respect to the constraints (see, for instance, the contemporary monograph [15]). For potential operator equations with side constraints, the direct variational methods and optimization principles are well developed tools that have been used in different problems (see [1], for instance). However all these methods fail for nonpotential operators, as arise in many applications, like second order elliptic equations with convective or asymmetric terms.

In this work we are motivated by two examples arising in an elastohydrodynamic lubrication problem and in a spatially aggregating population model, in which the presence of nonlinear diffusion and convection with a prescribed side condition, respectively, the load constraint and the total population, leads to a class of nonconvex side conditions. For both examples, we were unable to apply directly the classical variational methods, so that, developing a new technique based on convexity properties and on the continuous variation of a real parameter, which can be considered a generalized Lagrange multiplier for nonpotential and nondifferentiable problems, we are able to obtain sufficient conditions for their solvability.

In fact, our main result is valid in a rather general framework, namely for coercive pseudo-monotone operators with nonconvex constraints, that are given by level sets of convex functions, and is based on a continuous dependence property of an auxiliary family of elliptic variational inequalities (see [2], [9, Chapter 2.8] or [15, Chapter 54]). We give sufficient conditions for the existence, and in some cases also uniqueness, of solutions, that are found in the two examples of second order quasilinear elliptic equations and in two problems from applied sciences. 


\section{Elliptic pseudo-variational inequalities}

In the classical general framework of elliptic variational inequalities, (see [9], for instance) we consider the new class of problems of finding

$$
u \in K_{\Psi}:\langle A u, v-u\rangle \geq 0, \quad \forall v \in K_{\Psi}
$$

where we make the following assumptions: $K$ is a nonempty, closed, convex subset of a reflexive Banach space $V$ with the norm $\|\cdot\|$ and $V^{\prime}$ its dual; $A: K \subset V \rightarrow V^{\prime}$ is a pseudo-monotone operator and, for a given $I \in \mathbf{R}$, we set

$$
K_{\Psi}=\{v \in K: \Psi(v)=I\} .
$$

When $\Psi: V \rightarrow]-\infty,+\infty$ ] is a linear function, $K_{\Psi}$ is still a convex set and (1.1) is a well studied variational inequality. We are interested here in the case of a convex, lower semi-continuous function $\Psi(\Psi \not \equiv+\infty)$, therefore when $K_{\Psi}$ is the intersection of a convex set with a level set of a convex function. In this case, we call problem (1.1) an abstract elliptic pseudo-variational inequality.

By recalling the definition of the sub-differential $\partial \Psi(u)$ of $\Psi$ at $u$ and the generalized Lagrange multiplier rule (see [15], for instance), in the case $K=V$ we can formally rewrite the pseudo-variational inequality (1.1) as the problem of finding simultaneously $u \in V$ and a real number $\lambda \in \mathbf{R}$, such that

$$
A u+\lambda \partial \Psi(u) \ni 0 \text { in } V^{\prime}
$$

This remark suggests that we consider, for each $\lambda \in \mathbf{R}$, the auxiliary family of elliptic variational inequalities

$$
u_{\lambda} \in K:\left\langle A u_{\lambda}, v-u_{\lambda}\right\rangle+\lambda \Psi(v)-\lambda \Psi\left(u_{\lambda}\right) \geq 0, \forall v \in K
$$

for which we shall assume suitable assumptions to guarantee the respective existence and uniqueness of the solutions. In the case of a convex constraint $\Psi$ we consider (1.4) with $\lambda>0$, but the limit case $\lambda=0$ taking the form

$$
u_{0} \in K \cap \overline{\operatorname{Dom} \Psi}:\left\langle A u_{0}, v-u_{0}\right\rangle \geq 0, \forall v \in K \cap \overline{\operatorname{Dom} \Psi}
$$

has also interest. Here $\operatorname{Dom} \Psi=\{v \in V: \Psi(v)<+\infty\}$ is the effective domain of $\Psi$, $\widehat{\operatorname{Dom} \Psi}$ its closure in $V$, and we shall assume $K \cap \operatorname{Dom} \Psi \neq \emptyset$. We consider the following abstract framework for pseudo-monotone operators, i.e., for bounded nonlinear operators such that,

$$
\begin{gathered}
\text { if } u_{n} \rightarrow u \text { in } V-\text { weak and } \limsup _{n \rightarrow+\infty}\left\langle A u_{n}, u_{n}-u\right\rangle \leq 0, \text { then } \\
\qquad \liminf _{n \rightarrow+\infty}\left\langle A u_{n}, u_{n}-v\right\rangle \geq\langle A u, u-v\rangle, \forall v \in V .
\end{gathered}
$$


Assumption I. The pseudo-monotone operator $A$ is coercive in the sense that there exists a function $\alpha: \mathbf{R}^{+} \rightarrow[0,+\infty[$ with $\alpha(r) \rightarrow+\infty$ as $r \rightarrow+\infty$, such that, for any $v_{0} \in K \cap \operatorname{Dom} \Psi$ there is a constant $C_{0}>0$ and

$$
\left\langle A v, v-v_{0}\right\rangle \geq \alpha(\|v\|)\|v\|-C_{0}, \forall v \in K ;
$$

the convex function $\Psi: D o m \Psi \cap K \subset V \rightarrow \mathbf{R}$ is continuous for the weak topology of $V$.

Assumption $\mathrm{I}^{\prime}$. The pseudo-monotone operator $A$ is strongly coercive in the sense that, there exists a continuous function $\gamma$, strictly increasing from $[0,+\infty[$ to $[0,+\infty[$, with $\gamma(0)=0$ and $\gamma(r) \rightarrow+\infty$ as $r \rightarrow+\infty$, such that for any $v, w \in K \cap D o m \Psi$ :

$$
\langle A v-A w, v-w\rangle \geq\{\gamma(\|v-w\|)-\rho(v-w)\}\|v-w\|,
$$

where $\rho: V \rightarrow[0,+\infty[$ is a bounded function, satisfying $\rho(z) \rightarrow 0$ if $z \rightarrow 0$ in $V$-weak; $\Psi: K \subset V \rightarrow \mathbf{R}$ is a convex, lower semi-continuous function.

In particular, each one of these assumptions is a sufficient condition for the existence of at least one solution $u_{2}$ to (1.4), for each $\lambda \geq 0$ (see [9], or [15], for instance). We need also to assume the following strict inequality:

$$
I_{-} \equiv \inf _{v \in K \cap D o m \Psi} \Psi(v)<I_{+} \equiv \sup _{k \geq 0} \Psi\left(u_{2}\right)
$$

Theorem 1.1. Under Assumption I or $\mathbf{I}^{\prime}$ suppose that (1.4) $)_{\geq \geq 0}$ admits exactly one solution for every $\lambda \geq 0$ and (1.7) holds. Then for each

$$
\left.I \in] I_{-}, I_{+}\right]
$$

$\left(I_{-}<I<+\infty\right.$ if $\left.I_{+}=+\infty\right)$, there exists at least one solution $u_{I}$ to the pseudo-variational inequality (1.1).

Proof. If we show that the real function

$$
\phi:\left[0,+\infty\left[\ni \lambda \rightarrow \phi(\lambda)=\Psi\left(u_{\lambda}\right) \in\right] I_{-}, I_{+}\right]
$$

is such that:

(i) $\phi$ is continuous;

(ii) $\phi(\lambda) \rightarrow+\infty$ as $\lambda \rightarrow 0$ if $I_{+}=+\infty$;

(iii) $\phi(\lambda) \rightarrow I_{-}$if $\lambda \rightarrow+\infty$,

then there exists a $\lambda_{*} \in\left[0,+\infty\left[\right.\right.$ such that $\phi\left(\lambda_{*}\right)=I$ and the corresponding solution of $(1.4)_{\lambda_{*}} u_{\lambda_{2}}$ will solve also (1.1), proving the theorem. 
(i) We start with the continuity of $\phi$, which is essentially the continuous dependence property $\left[0,+\infty\left[\ni \lambda \rightarrow u_{\lambda} \in V\right.\right.$ for the weak (resp. strong) topology in case I (resp. case $I^{\prime}$ ). From (1.4), for $\lambda>0$ and using the coercivity (1.5) (or (1.6) which is stronger) we have

$$
\begin{aligned}
\lambda \Psi\left(v_{0}\right) & \geq \lambda \Psi\left(u_{\lambda}\right)+\left\langle A u_{\lambda}, u_{\lambda}-v_{0}\right\rangle \\
& \geq\left\{\alpha\left(\left\|u_{\lambda}\right\|\right)-\lambda C_{1}\right\}\left\|u_{\lambda}\right\|-C_{0}-\lambda C_{2},
\end{aligned}
$$

since, by convexity of $\Psi$, we have $\Psi\left(u_{\lambda}\right) \geq-C_{1}\left\|u_{\lambda}\right\|-C_{2}$, for $C_{1}, C_{2} \in \mathbf{R}$.

From (1.8), if we take any convergent sequence $\lambda_{n} \underset{n \rightarrow \infty}{\longrightarrow} \lambda \in\left[0,+\infty\left[, \lambda_{n}>0\right.\right.$, we immediately conclude that $u_{\lambda_{n}}$ is such that $\left\|u_{\lambda_{n}}\right\| \leq C$ (independently of $n \rightarrow \infty$ ) and there exists $u_{*} \in K \cap \overline{D o m} \Psi$, such that, for a subsequence still denoted by $n \rightarrow \infty$, $u_{\lambda_{n}} \rightarrow u_{*}$ in $V$-weak. If $\lambda>0$ or $\lambda=0$ and $I_{+}<+\infty$, by the lower semi-continuity of $\Psi$ we have

$$
\liminf _{n \rightarrow \infty} \lambda_{n} \Psi\left(u_{\lambda_{n}}\right) \geq \lambda \Psi\left(u_{*}\right)
$$

and

$$
\underset{n \rightarrow \infty}{\limsup }\left\langle A u_{\lambda_{n}}, u_{\lambda_{n}}-u_{\star}\right\rangle \leq \limsup _{n \rightarrow \infty}\left[\lambda_{n} \Psi\left(u_{*}\right)-\lambda_{n} \Psi\left(u_{\lambda_{n}}\right)\right] \leq 0
$$

Since $A$ is a pseudo-monotone operator, it follows that

$$
\liminf _{n \rightarrow \infty}\left\langle A u_{\lambda_{n}}, u_{\lambda_{n}}-v\right\rangle \geq\left\langle A u_{*}, u_{*}-v\right\rangle, \forall v \in K
$$

and passing to the limit in (1.4) $)_{\lambda_{n}}$, by the uniqueness of the limit problem (1.4) $)_{\lambda}$, we easily conclude that $u_{*}=u_{\lambda}$, showing the weak continuity in the first case I. If (1.6) holds, letting $v=u_{\lambda}$ in (1.4) $)_{\lambda_{n}}$ and $v=u_{\lambda_{n}}$ in (1.4), after subtraction, we obtain

$$
\begin{gathered}
\left(\lambda-\lambda_{n}\right)\left[\Psi\left(u_{\lambda_{n}}\right)-\Psi\left(u_{\lambda}\right)\right] \geq\left\langle A u_{\lambda}-A u_{\lambda_{n}}, u_{\lambda}-u_{\lambda_{n}}\right\} \\
\geq\left\{\gamma\left(\left\|u_{\lambda}-u_{\lambda_{n}}\right\|\right)-\rho\left(u_{\lambda}-u_{\lambda_{n}}\right)\right\}\left\|u_{\lambda}-u_{\lambda_{n}}\right\|
\end{gathered}
$$

and, since $u_{\lambda_{n}} \rightarrow u_{\lambda}$, when $\lambda_{n} \rightarrow \lambda$, we conclude also $\left\|u_{\lambda}-u_{\lambda_{n}}\right\| \rightarrow 0$. Then, $\Psi$ being a convex function bounded in a neighbourhood of the point $u_{\lambda}$, it is then continuous at $u_{\lambda}$ and the continuity of $\phi$ follows.

(ii) If $\lambda \rightarrow 0$ and $\Psi\left(u_{0}\right)=+\infty, u_{0}$ being the unique solution to (1.4), from (1.8), the sequence $u_{\lambda}$ is bounded in $V$, independently of $0<\lambda<1$, and we may assume $u_{\lambda} \rightarrow u$, in $V$-weak, for some $u_{*} \in \overline{\operatorname{Dom} \Psi} \cap K$. For $\epsilon>0$, take $u_{\epsilon} \in \operatorname{Dom} \Psi \cap K$ such that $\left\|u_{*}-u_{\epsilon}\right\|<\epsilon$, and we obtain, with $v=u_{\epsilon}$ in (1.4) $\lambda$ 


$$
\begin{gathered}
\limsup _{\lambda \rightarrow 0}\left(A u_{\lambda}, u_{\lambda}-u_{*}\right) \leq C \epsilon+\limsup _{\lambda \rightarrow 0}\left(A u_{\lambda}, u_{\lambda}-u_{\epsilon}\right) \\
\leq C \epsilon+\limsup _{\lambda \rightarrow 0}\left\{\lambda \Psi\left(u_{\epsilon}\right)-\lambda \Psi\left(u_{\lambda}\right)\right\} \leq C \epsilon,
\end{gathered}
$$

since $\liminf _{\lambda \rightarrow 0} \lambda \Psi\left(u_{\lambda}\right) \geq 0$. Since $\epsilon$ is arbitrary, we can use again the pseudomonotonicity of $A$ and take the $\liminf _{\lambda \rightarrow 0}$ in (1.4) $)_{\lambda>0}$ with $v \in \operatorname{Dom} \Psi \cap K$ in order to conclude

$$
\left\langle A u_{*}, v-u_{*}\right\rangle \geq 0
$$

first for any $v \in \operatorname{Dom} \Psi \cap K$, and by density, also for all $v \in \overline{\operatorname{Dom} \Psi} \cap K$. Consequently $u_{*}=u_{0}$ and $\liminf _{\lambda \rightarrow 0} \Psi\left(u_{\lambda}\right) \geq \Psi\left(u_{0}\right)=+\infty$ yields $\phi(\lambda) \rightarrow+\infty$ when $\lambda \rightarrow 0$ and $I_{+}=+\infty$.

(iii) Using the coercivity (1.5), from (1.4), for $\lambda>0$ we obtain

$$
\begin{aligned}
\Psi\left(v_{0}\right) & \geq \Psi\left(u_{\lambda}\right)+\frac{1}{\lambda}\left\{\alpha\left(\left\|u_{\lambda}\right\|\right)\left\|u_{\lambda}\right\|-C_{0}\right\} \\
& \geq \Psi\left(u_{\lambda}\right)+O\left(\frac{1}{\lambda}\right) \text { as } \lambda \rightarrow+\infty
\end{aligned}
$$

Consequently we obtain

$$
\Psi(v) \geq \limsup _{\lambda \rightarrow+\infty} \Psi\left(u_{\lambda}\right), \forall v \in \operatorname{Dom} \Psi \cap K
$$

Hence

$$
\liminf _{\lambda \rightarrow+\infty} \Psi\left(u_{\lambda}\right) \geq \inf _{v \in K \cap D o m \Psi} \Psi(v)=I_{-} \geq \limsup _{\lambda \rightarrow+\infty} \Psi\left(u_{\lambda}\right)
$$

which yields

$$
I_{-}=\lim _{\lambda \rightarrow+\infty} \Psi\left(u_{\lambda}\right)=\lim _{\lambda \rightarrow+\infty} \phi(\lambda)
$$

concluding the proof.

Corollary 1.2. Assuming (1.7) and the Assumption I or $\mathbf{I}^{\prime}$, if the operator $A$ is also strictly monotone, i.e.,

$$
(A v-A w, v-w)>0, \forall v, w \in K \cap \operatorname{Dom} \Psi, w \neq v
$$

then there exists a unique solution $u_{l}$ to (1.1) for each $\left.\left.I \in\right] I_{-}, I_{+}\right]\left(I<+\infty\right.$ if $\left.I_{+}=+\infty\right)$. 
Proof. It suffices to remark that (1.9) implies the uniqueness for the problems (1.4),,$\forall \lambda \geq 0$, as well as for (1.1). Indeed if $u_{1}$ and $u_{2}$ are two different solutions of (1.1), letting $v=u_{1} \in K_{\Psi}$ and $v=u_{2} \in K_{\Psi}$, respectively, in (1.1) for $u_{2}$ and for $u_{1}$, after subtraction we obtain

$$
\left\langle A u_{1}-A u_{2}, u_{1}-u_{2}\right\rangle \leq 0
$$

and therefore $u_{1}=u_{2}$.

Remark 1.3. Although the strict monotonicity of the operator $A$ is a sufficient condition it is not a necessary one for uniqueness, as we shall show in the next section.

Analogously we may solve the pseudo-variational inequality (1.1) with a concave function $\Psi$ in the definition (1.2). In fact, since $\lambda \Psi$ is then a convex function for $\lambda<0$ it is sufficient to consider (1.4) $)_{\lambda}$ with negative $\lambda$ and replace the condition (1.7) by

$$
J_{-} \equiv \inf _{\lambda \leq 0} \Psi\left(u_{\lambda}\right)<J_{+} \equiv \sup _{v \in K \cap D o m \Psi} \Psi(v) .
$$

Theorem 1.4. Assuming (1.10) and the Assumption $\mathbf{I}$ or $\mathbf{I}^{\prime}$, if (1.4) $)_{\lambda \leq 0}$ admits exactly one solution for every $\lambda \leq 0$ with $\Psi$ concave, then for each $I \in\left[J_{-}, J_{+}\left[\left(J_{+}>I>-\infty\right.\right.\right.$ if $J_{-}=-\infty$ ) there exists at least one solution $u_{1}$ to (1.1) with $\Psi$ concave in (1.2). If, in addition, $A$ is strictly monotone, the solution of $(1.1)$ is unique.

The strict inequality in (1.7) (resp. (1.10), for the concave case) are sufficient to guarantee that $K_{\psi}$, defined in (1.2) is non-empty for $\left.\left.I \in\right]-I_{-}, I_{+}\right]$(resp. $I \in\left[J_{-}, J_{+}[\right.$), but the determination of. $I_{+}$(resp. $J_{-}$) depends on $A, K$ and $\Psi$ through the auxiliary problems $(1.4)_{\lambda}$ or $(1.4)_{0}$. For instance, $I_{0}=\Psi\left(u_{0}\right)$, where $u_{0}$ solves $(1.4)_{0}$, is a lower (resp. upper) estimate for $I_{+}$(resp. $J_{-}$), but it can be its value exactly if additional properties are known, as in the monotone case.

Suppose the Banach space $V$ has a partial order relation where $u \leq v$ is equivalent to $(u-v)^{+}=0$, for which we can define, for every $u, v \in K$

$$
u \vee v=u+(v-u)^{+} \in K \text { and } u \wedge v=v-(v-u)^{+} \in K
$$

and assume, for the convex function $\Psi$,

$$
\begin{aligned}
\Psi\left(v_{1}\right)+\Psi\left(v_{2}\right) & \geq \Psi\left(v_{1} \vee v_{2}\right)+\Psi\left(v_{1} \wedge v_{2}\right), \forall v_{1}, v_{2} \in \operatorname{Dom} \Psi, \\
\Psi\left(v_{2}\right) & \geq \Psi\left(v_{1}\right) \text { if } v_{2} \geq v_{1} \quad(\Psi \text { is increasing })
\end{aligned}
$$

and the operator $A$ is strictly $T$-monotone in the sense that 
CLASS OF ELLIPTIC PROBLEMS WITH NONCONVEX CONSTRAINTS 339

$$
\left(A v-A w,(v-w)^{+}\right\rangle>0, \forall v, w \in K: 0 \neq(v-w)^{+}
$$

in particular, $\boldsymbol{A}$ is strictly monotone.

Proposition 1.5. Assuming (1.11)-(1.14), if $u_{1}=u_{\lambda_{1}}$ and $u_{2}=u_{\lambda_{2}}$ denote the solutions of $\left(1.4_{\lambda_{1}}\right)$ and $(1.4)_{\lambda_{2}}$, respectively,

$$
\text { if } \lambda_{1} \geq \lambda_{2} \geq 0 \text { then } u_{2} \geq u_{1} \text {, }
$$

and we can take $I_{+}=\Psi\left(u_{0}\right)$ in (1.7), where $u_{0}$ solves the variational inequality $(1.4)_{0}$, under the Assumption I.

Proof. Taking $v=u_{1} \vee u_{2}=u_{2}+\left(u_{1}-u_{2}\right)^{+}$in $(1.4)_{\lambda_{2}}$ and $v=u_{1} \wedge u_{2}=u_{1}-\left(u_{1}-u_{2}\right)^{+}$ in (1.4) $\lambda_{\lambda_{1}}$, for $\lambda_{1}>\lambda_{2}>0$, by subtraction we obtain

$$
\begin{gathered}
\left\langle A u_{1}-A u_{2},\left(u_{1}-u_{2}\right)^{+}\right\rangle \leq \lambda_{2} \Psi\left(u_{1} \vee u_{2}\right)-\lambda_{2} \Psi\left(u_{2}\right)+ \\
+\lambda_{1} \Psi\left(u_{1} \wedge u_{2}\right)-\lambda_{1} \Psi\left(u_{1}\right) .
\end{gathered}
$$

Adding and subtracting $\lambda_{2}\left[\Psi\left(u_{1}\right)-\Psi\left(u_{1} \wedge u_{2}\right)\right]$, the right-hand side yields, by (1.12) and (1.13),

$$
\begin{gathered}
\lambda_{2}\left[\Psi\left(u_{1} \vee u_{2}\right)+\Psi\left(u_{1} \wedge u_{2}\right)-\Psi\left(u_{2}\right)-\Psi\left(u_{1}\right)\right] \\
+\left(\lambda_{2}-\lambda_{1}\right)\left[\Psi\left(u_{1}\right)-\Psi\left(u_{1} \wedge u_{2}\right)\right] \leq 0
\end{gathered}
$$

which combined with (1.14) implies $\left(u_{1}-u_{2}\right)^{+}=0$, i.e., $u_{2} \geq u_{1}$. By (1.13) it follows that when $\lambda \searrow 0, u_{\lambda} \rightarrow u_{0}$ in $V$-weak and $\Psi\left(u_{\lambda}\right)$ is decreasing in $\lambda$, and therefore increases as $\lambda$ decreases to 0 . Therefore we conclude $I_{+}=\Psi\left(u_{0}\right)$ from

$$
\Psi\left(u_{0}\right) \leq \liminf _{\lambda>0} \Psi\left(u_{\lambda}\right) \leq \underset{\lambda \backslash 0}{\lim \sup } \Psi\left(u_{\lambda}\right) \leq \Psi\left(u_{0}\right) \leq+\infty
$$

Remark 1.6. As in Theorem 1.4, for a concave decreasing function $\Psi$ satisfying (1.12) with the reverse inequality, we may take $J_{-}=\Psi\left(u_{0}\right)$, since, analogously to the Proposition 1.5, we can show that $\lambda_{1} \leq \lambda_{2} \leq 0$ yields $u_{\lambda_{2}} \geq u_{\lambda_{1}}$.

\section{Applications to nonlinear elliptic problems}

In this section we exemplify the general approach of Section 1 in two classes of nonpotential elliptic partial differential operators of second order,

$$
A_{1} u=-\Delta u+b(x, u, \nabla u)
$$




$$
A_{p} u=-\nabla \cdot\left(|\nabla u|^{p-2} \nabla u+B(x, u)\right)+\gamma(x, u)
$$

in a bounded open domain $\Omega \subset \mathbf{R}^{n}, n \geq 1$, where, for simplicity, we consider only homogeneous Dirichlet boundary conditions. We shall consider classic convex constraints in the definition of the convex set $K$ and nonclassic nonconvex constraints through special cases of $\Psi$, without searching for the most general examples.

2.2. - First example. Let $V=W_{0}^{1, p}(\Omega)$ denote the usual Sobolev space with norm $\|v\|_{p}=\left(\int_{\Omega}|\nabla v|^{p}\right)^{1 / p}, 1<p<\infty$, and define $\Psi: H_{0}^{1}(\Omega)=W_{0}^{1,2}(\Omega) \rightarrow[0, \infty[$ by

$$
\Psi(v)=\int_{\omega}|\nabla v| d x, \quad \omega \subset \Omega
$$

where $\omega$ is an arbitrary nonempty open subset of $\Omega$. For a given $I>0$ consider the elliptic pseudo-variational inequality for $A_{1}$ given by (2.1):

$$
\begin{gathered}
u \in K_{I}=\left\{v \in H_{0}^{1}(\Omega): \int_{\omega}|\nabla v| d x=I\right\} \\
\int_{\Omega} \nabla u \cdot \nabla(v-u)+\int_{\Omega} b(x, u, \nabla u)(v-u) \geq 0, \forall v \in K_{I} .
\end{gathered}
$$

Here the nonlinear term is given by a measurable function $b=b(x, u, \xi): \Omega \times$ $\mathbf{R} \times \mathbf{R}^{n} \rightarrow \mathbf{R}$, under the assumptions of continuity in the variables $u$ and $\xi$, and satisfying the conditions, for a.e. $x \in \Omega, \forall u, v \in \mathbf{R}$ and $\forall \xi, \eta \in \mathbf{R}^{n}$ :

$$
\begin{gathered}
(b(x, u, \xi)-b(x, v, \xi))(u-v) \geq 0, \\
|b(x, u, \xi)-b(x, u, \eta)| \leq b_{1}(x, u)|\xi-\eta|, \\
|b(x, u, \xi)| \leq f_{0}(x),
\end{gathered}
$$

where $f_{0} \in L^{2}(\Omega)$ and $b_{1}(x, u(x)) \in L^{s}(\Omega), s=n \geq 3$ or $s>n=1,2$, for each $u \in H_{0}^{1}(\Omega)$.

For each $\lambda>0$, we consider the auxiliary variational inequality

$$
\begin{aligned}
u_{\lambda} \in H_{0}^{\mathrm{l}}(\Omega): & \int_{\Omega} \nabla u_{\lambda} \cdot \nabla\left(v-u_{\lambda}\right)+\int_{\Omega} b\left(x, u_{\lambda}, \nabla u_{\lambda}\right)\left(v-u_{\lambda}\right) \\
& +\lambda \int_{\omega}|\nabla v|-\lambda \int_{\omega}\left|\nabla u_{\lambda}\right| \geq 0, \forall v \in H_{0}^{1}(\Omega) .
\end{aligned}
$$

Proposition 2.1. Under the assumptions (2.6)-(2.8) there exists a unique solution $u_{2}$ to $(2.9)_{\lambda}, \forall \lambda>0$, as well as a unique 
CLASS OF ELLIPTIC PROBLEMS WITH NONCONVEX CONSTRAINTS 341

$$
u_{0} \in H_{0}^{1}(\Omega):-\Delta u_{0}+b\left(x, u_{0}, \nabla u_{0}\right)=0 \text { in } \Omega
$$

which is the strong limit in $H_{0}^{1}(\Omega)$ of $u_{\lambda}$ as $\lambda \rightarrow 0$.

Proof. The existence is an immediate consequence of the fact that the associated operator $A_{1}: H_{0}^{1}(\Omega) \rightarrow H^{-1}(\Omega)$ is pseudo-monotone and strongly coercive. The uniqueness follows by an adaptation of an argument of Trudinger (see [13] and [5] for the case $(2.9)_{0}$ ): suppose for contradiction, that for two different solutions $u$ and $w$ to $(2.9)_{\lambda}$, we have $M=\sup _{\Omega}(u-w)>0$; set $z_{k}=(u-w-k)^{+}$for $0<k<M \leq+\infty$ and take $v_{u}=u-z_{k}$ in (2.9) $)_{\lambda}$ for $u$ and $v_{w}=w+z_{k}$ in (2.9), for $w$; since $\nabla v_{u}=\nabla u$ and $\nabla v_{w}=\nabla w$ a.e. in $\{u \leq w+k\}$ and $\nabla v_{u}=\nabla w$ and $\nabla v_{w}=\nabla u$ a.e. in $\{u>w+k\}$, by subtraction we obtain

$$
\begin{aligned}
\int_{\Omega_{k}}|\nabla(u-w)|^{2}=\int_{\Omega}\left|\nabla z_{k}\right|^{2} & \leq \int_{\Omega} b_{1}\left|\nabla z_{k}\right| z_{k}=\int_{\Omega_{k}} b_{1}\left|\nabla z_{k}\right| z_{k} \\
& \leq\left\|b_{1}\right\|_{L^{3}\left(\Omega_{k}\right)}\left\|\nabla z_{k}\right\|_{L^{2}(\Omega)}\left\|z_{k}\right\|_{L^{\frac{2 s}{s^{2}}}(\Omega)}
\end{aligned}
$$

where $\Omega_{k}=\left\{x \in \Omega: z_{k}>0,\left|\nabla z_{k}\right|>0\right\}$; therefore, using the Sobolev inequality, for some $C_{*}>0$ we deduce from $(2.10)$

$$
1 \leq C_{*}\left\|b_{1}\right\|_{L^{\prime}\left(\Omega_{k}\right)}, \forall k: 0<k<M \leq+\infty,
$$

which is impossible, since meas $\left(\Omega_{k}\right) \rightarrow 0$ as $k \rightarrow M$. Hence $M=0$ and $u \leq w$. Analogously we conclude $w \leq u$ and the uniqueness follows, as well as, the continuous dependence with respect to $\lambda \in[0,+\infty[$.

We may now apply Theorem 1.1 to solve (2.4)-(2.5). First we observe that we have

$$
\begin{gathered}
I_{-} \equiv \inf _{v \in H_{0}^{\mathrm{l}}(\Omega)} \int_{\omega}|\nabla v| d x=0, \\
I_{+} \equiv \sup _{\lambda \geq 0} \int_{\omega}\left|\nabla u_{\lambda}\right| d x \geq \int_{\omega}\left|\nabla u_{0}\right| d x=I_{0}\left(u_{0}\right),
\end{gathered}
$$

and we must guarantee that $I_{0}\left(u_{0}\right)>0$, where $u_{0}$ is the solution of $(2.9)_{0}$. A sufficient condition is given by the following additional assumption on $b$ :

$$
b(x, c, 0) \neq 0, \forall c \in \mathbf{R} \text {, a.e. } x \in \omega .
$$

Theorem 2.1. Under the assumptions (2.6), (2.7), (2.8) and (2.11), for each $\left.I \in] 0, I_{0}\right]$, where $I_{0}=\int_{\omega}\left|\nabla u_{0}\right| d x$, there exists at least one solution to (2.4)-(2.5). 
Proof. In fact, by Theorem 1.1, it remains to show that $I_{0}>0$ to fulfil the assumption (1.7). Assume to the contrary that $I_{0}=0$; then $\left|\nabla u_{0}\right|=0$ a.e. in the open set $\omega$ and $u_{0}=$ constant in each connected open component $\omega_{j} \subset \omega$; hence also $\Delta u_{0}=0$ in each $\omega_{j}$, which is a contradiction with $(2.9)_{0}$ and (2.11).

Remark 2.1. We know that the solution $u$ to (2.4)-(2.5) also solves (2.9), for a certain $\lambda_{I} \geq 0$. Hence, if $\omega=\Omega$ and $|\nabla u|>0$ a.e. in $\Omega$, we may also conclude that $u$ solves in $H_{0}^{1}(\Omega)$ the equation

$$
-\nabla \cdot\left(\nabla u+\lambda_{I} \frac{\nabla u}{|\nabla u|}\right)+b(u, \nabla u)=0 \text { in } \Omega
$$

with the nonconvex constraint $\int_{\Omega}|\nabla u| d x=I$. We remark these type of equations arise in the stationary flow of Bingham fluids (see [4], for instance).

Remark 2.2. We do not know if, in general, the solution to (2.4)-(2.5) is unique, except in the special case when $b$ does not depend on the gradient, which is then a special case of Corollary 1.2. However in this case, a direct optimization approach is possible, since it corresponds to the minimization of the functional

$$
J(u)=\int_{\Omega}\left(\frac{1}{2}|\nabla u|^{2}+\int_{0}^{u} b(x, s) d s\right) d x
$$

in the closed subset $K_{I}$ defined by (2.4) (see [15], for instance).

Remark 2.3. It is also possible to extend our results to the case where the unnecessary boundedness restriction (2.8) is replaced by an appropriate growth assumption or by a condition of the type $u b(x, u, \xi) \geq 0$. Also in the linear case, we can consider an elliptical operator of the form

$$
A_{1} u=-\sum_{i j} \frac{\partial}{\partial x_{i}}\left(a_{i j} \frac{\partial u}{\partial x_{j}}+e_{i} u+f_{i}\right)+\sum_{i} b_{i} \frac{\partial u}{\partial x_{i}}+c u+f_{0}
$$

with the restriction $c-\sum_{i} \frac{\partial e_{i}}{\partial x_{i}} \geq 0$ (see [13] and its bibliography, for instance).

2.2 - Second example. Let $\psi: \Omega \times[0,+\infty[\rightarrow[0,+\infty[$, be a convex integrand, i.e., a measurable function, such that,

$$
\begin{gathered}
s \rightarrow \psi(x, s) \text { is convex, a.e. } x \in \Omega, \text { and } \\
\Psi(v)=\int_{\Omega} \psi(x, v(x)) d x<\infty, \text { for each } v \in W_{0}^{1, p}(\Omega), v \geq 0 .
\end{gathered}
$$

For $p \geq 2$, we shall define 


\section{CLASS OF ELLIPTIC PROBLEMS WITH NONCONVEX CONSTRAINTS 343}

$$
K=\operatorname{Dom} \Psi=\left\{v \in W_{0}^{1, p}(\Omega): v \geq 0 \text { a.e. in } \Omega\right\}
$$

For a given $J>0$, we consider now the following constrained problem for the operator $A_{p}$ given in (2.2):

$$
\begin{gathered}
u \in K_{J}=\left\{v \in K: \int_{\Omega} \psi(x, v(x)) d x=J\right\} \\
\int_{\Omega}\left(|\nabla u|^{p-2} \nabla u+B(x, u)\right) \cdot \nabla(v-u)+\int_{\Omega} \gamma(x, u)(v-u) \geq 0, \forall v \in K_{J} .
\end{gathered}
$$

Here $B(x, u): \Omega \times \mathbf{R} \rightarrow \mathbf{R}^{n}$ and $\gamma(x, u): \Omega \times \mathbf{R} \rightarrow \mathbf{R}$ are given measurable functions, continuous in the variable $u$ and such that, for each $u \in W_{0}^{1, p}(\Omega), B(u) \in\left[L^{p}(\Omega)\right]^{n}$ and $\gamma(u) \in L^{p^{*}}(\Omega)$, where $p^{*}=1$ if $p>n, p^{*}>1$ if $p=n$ or $p^{*}=\frac{p n}{n(p-1)+p}$ if $2 \leq p<n$, and the operator $A_{p}: W_{0}^{1, p}(\Omega) \rightarrow W^{-1, p^{\prime}}(\Omega)$ is a bounded, pseudo-monotone operator satisfying the coercivity condition (1.6). In addition, we suppose there exists a $g \in L^{p^{\prime}}(\Omega)$, $p^{\prime}=p /(p-1)$, such that for a.e. $x \in \Omega$ and all $u, v \in \mathbf{R}$

$$
|B(x, u)-B(x, v)| \leq g(x) \omega(|u-v|), \text { with } \int_{0^{+}} \frac{d s}{\omega^{p^{\prime}}(s)}=+\infty .
$$

In particular, since we consider $p \geq 2$, the condition (2.17) is satisfied if $B$ is Hölder continuous with exponent $\alpha, 1 / 2 \leq 1 / p^{\prime} \leq \alpha \leq 1$.

Finally, following [3], we shall also impose

$$
u \mapsto \gamma(x, u) \text { is strictly increasing, a.e. } x \in \Omega
$$

or, in the special case arising in applications,

$$
p=2, u \mapsto \gamma(x, u) \text { is nondecreasing, and }
$$

$\exists \xi \in \mathbf{R}^{n} \backslash\{0\}: u \mapsto \xi \cdot B(x, u)$ is monotone, for a.e. $x \in \Omega$.

For every $\lambda \geq 0$, we consider the family of auxiliary well-posed variational inequalities

$$
\begin{aligned}
u_{\lambda} \in K: \int_{\Omega}\left[\left|\nabla u_{\lambda}\right|^{p-2} \nabla u_{\lambda}\right. & \left.+B\left(x, u_{\lambda}\right)\right] \cdot \nabla\left(v-u_{\lambda}\right) d x+\int_{\Omega} \gamma\left(x, u_{\lambda}\right)\left(v-u_{\lambda}\right) d x \\
& +\lambda \int_{\Omega} \psi(x, v) d x-\lambda \int_{\Omega} \psi\left(x, u_{\lambda}\right) d x \geq 0, \forall v \in K
\end{aligned}
$$

Proposition 2.2. Under the above assumptions, namely (2.12)-(2.14), (1.5), (2.17) and (2.18) or (2.19), the map 


$$
\left[0,+\infty\left[\ni \lambda \mapsto u_{\lambda} \in K \text { is continuous in } W_{0}^{1, p}(\Omega)\right.\right.
$$

where $u_{\lambda}$ is the unique solution of $(2.20)_{\lambda}$.

Proof. The existence is a consequence of general results for pseudo-monotone variational inequalities of the type (1.4), (see [9, p. 251, for instance]), while the strong continuity can be obtained, as in Theorem 1.1, once the uniqueness of the solution is established. Note that, in fact, $A_{p}$ is strongly coercive for the continuous dependence.

The uniqueness for $(2.20)_{\lambda}$ in the case $\lambda=0$ was shown in Theorem 2.1 of [3] for the case (2.18) and in Theorem 5.2 of [3] for the case (2.19). Also for the case $\lambda>0$, we can easily extend the arguments of [3] by controlling the additional terms given by $\Psi$ in the following manner: suppose we have two solutions $u_{1}$ and $u_{2}$ of $(2.20)_{\lambda}$, and consider the nonnegative Lipschitz function $F^{\varepsilon}$ defined by

$$
F^{e}(t)= \begin{cases}\frac{1}{t(\varepsilon)} \int_{\varepsilon}^{t} \frac{d s}{\omega^{p^{\prime}}(s)} & \text { for } t>\varepsilon>0 \\ 0 & \text { for } t \leq \varepsilon\end{cases}
$$

where we have set $t(\varepsilon)=\int_{\varepsilon}^{+\infty} d s / \omega^{p^{\prime}}(s)<+\infty$ without loss of generality; for an arbitrary $\eta \in C^{l}(\bar{\Omega}), \eta \geq 0$, choose $\delta>0$ sufficiently small, such that

$$
v_{1}=u_{1}+\delta \eta F^{e}\left(u_{2}-u_{1}\right) \in K \text { and } v_{2}=u_{2}-\delta \eta F^{\ell}\left(u_{2}-u_{1}\right) \in K
$$

taking these two functions, respectively, in (2.20), for $u_{1}$ and for $u_{2}$, we have

$$
\left\langle A u_{1}-A u_{2}, \delta \eta F^{e}\left(u_{2}-u_{1}\right)\right\rangle \geq \lambda \int_{\Omega}\left\{\psi\left(u_{1}\right)-\psi\left(v_{1}\right)+\psi\left(u_{2}\right)-\psi\left(v_{2}\right)\right\} d x \equiv \Lambda
$$

if $\Lambda \geq 0$, then, exactly as in the proofs of Theorems 2.1 and 5.2 of [3], we may conclude that $u_{2} \leq u_{1}$ a.e. in $\Omega$, and changing the role of $u_{1}$ and $u_{2}$, we conclude that $u_{\lambda}$ is unique.

The new remark is, in fact, the observation that from the definitions (2.22), there exists a function $\beta=\beta(x)$, such that:

$$
\begin{gathered}
0 \leq \beta(x) \leq 1 \text { a.e. } x \in \Omega ; \beta(x)=0 \text { if } u_{2}(x) \leq u_{1}(x) \\
v_{1}=u_{1}+\beta\left(u_{2}-u_{1}\right) ; \quad v_{2}=u_{2}-\beta\left(u_{2}-u_{1}\right)
\end{gathered}
$$

and, using the convexity of $\lambda \psi$, we may conclude

$$
\begin{aligned}
\Lambda & =\lambda \int_{\Omega}\left\{\psi\left(u_{1}\right)-\psi\left((1-\beta) u_{1}+\beta u_{2}\right)+\psi\left(u_{2}\right)-\psi\left(\beta u_{1}+(1-\beta) u_{2}\right)\right\} d x \\
& \geq \lambda \int_{\Omega}\left\{\psi\left(u_{1}\right)-(1-\beta) \psi\left(u_{1}\right)-\beta \psi\left(u_{2}\right)+\psi\left(u_{2}\right)-\beta \psi\left(u_{1}\right)-(1-\beta) \psi\left(u_{2}\right)\right\} d x=0 .
\end{aligned}
$$


Proposition 2.3. If, in addition to the assumptions of Proposition 2.2, we suppose that

$$
s \mapsto \psi(x, s) \text { is nondecreasing, a.e. } x \in \Omega,
$$

then the map (2.21) is nonincreasing, i.e.,

$$
\text { if } \lambda_{2} \geq \lambda_{1} \geq 0 \text { then } u_{\lambda_{1}} \geq u_{\lambda_{2}} \text { a.e. in } \Omega \text {. }
$$

Proof. Setting $u_{1}=u_{\lambda_{1}}$ and $u_{2}=u_{\lambda_{2}}$, we can repeat the argument of the proof in Proposition 2.2, observing now that the assumptions imply also

$$
\begin{aligned}
\Lambda & \equiv \int_{\Omega}\left\{\lambda_{1}\left[\psi\left(u_{1}\right)-\psi\left((1-\beta) u_{1}+\beta u_{2}\right)\right]+\lambda_{2}\left[\psi\left(u_{2}\right)-\psi\left(\beta u_{1}+(1-\beta) u_{2}\right)\right]\right\} d x \\
& \geq \int_{\Omega}\left\{\lambda_{1} \beta\left[\psi\left(u_{1}\right)-\psi\left(u_{2}\right)\right]+\lambda_{2} \beta\left[\psi\left(u_{2}\right)-\psi\left(u_{1}\right)\right]\right\} d x \\
& =\int_{\left(u_{2}>u_{1}\right\}} \beta\left(\lambda_{1}-\lambda_{2}\right)\left[\psi\left(u_{1}\right)-\psi\left(u_{2}\right)\right] d x \geq 0,
\end{aligned}
$$

since we have $\beta(x)=0$ if $u_{2}(x) \leq u_{1}(x)$.

We are now in the situation of Theorem 1.1, provided we add the assumption

$$
I_{0} \equiv \int_{\Omega} \psi\left(x, u_{0}(x)\right) d x>\inf _{v \in K} \int_{\Omega} \psi(x, v(x)) d x \equiv I_{-} \geq 0
$$

where $u_{0}$ denotes the unique solution to $(2.20)_{0}$ with $\lambda=0$. Notice that

$$
I_{+} \equiv \sup _{\lambda \geq 0} \int_{\Omega} \psi\left(x, u_{\lambda}(x)\right) d x \geq I_{0}
$$

where $u_{\lambda}$ denotes the solution to $(2.20)_{\lambda}$ and from the Proposition 2.3 we have $I_{0}=I_{+}$ if $\psi$ is nondecreasing in $u$, in which case also $I_{-}=\int_{\Omega} \psi(x, 0) d x$. Then we have proved the following existence theorem.

Theorem 2.2. Under the preceding assumptions, namely (2.12)-(2.14), (1.15), (2.17), (2.18) (or (2.19)) and (2.26), there are numbers $I_{+}>I_{-} \geq 0$ given by (2.26) and (2.27), such that, for each $\left.J \in] I_{-}, I_{+}\right]$, there exists at least one solution to the pseudo-variational inequality (2.15)-(2.16).

Remark 2.4. If, for instance, $\psi(x, s)$ is differentiable in $s$, the solution $u_{s}$ obtained in Theorem 2.2 , solving $(2.20)_{\lambda}$, for a certain $\lambda_{s} \geq 0$, also solves in $W_{0}^{1, p}(\Omega)$ the unilateral problem 


$$
\begin{gathered}
u \geq 0,-\nabla \cdot\left[|\nabla u|^{p-2} \nabla u+B(x, u)\right]+\gamma(x, u)+\lambda_{J} \frac{\partial \psi}{\partial s}(x, u) \geq 0 \text { in } \Omega, \\
\left(-\nabla \cdot\left[|\nabla u|^{p-2} \nabla u+B(x, u)\right]+\gamma(x, u)+\lambda_{J} \frac{\partial \psi}{\partial s}(x, u), u\right\rangle=0
\end{gathered}
$$

subject to the nonconvex constraint $\int_{\Omega} \psi(x, u) d x=J$.

Remark 2.5. Except in the case of $B$ independent of $u$, we were unable to prove the uniqueness for problem (2.15)-(2.16), since the test functions of (2.22) are not admissible in $K_{J}$, which is not a convex set in general. However the existence result can be extended to more general situations, for elliptic operators of the form

$$
A u=-\nabla \cdot a(x, u, \nabla u)+\gamma(x, u),
$$

for appropriate structural conditions on $a(x, u, \nabla u)$, and also for more general convex subsets $K \subset W^{1, p}(\Omega)$, with nonzero obstacles or with more general boundary conditions (see [3], for instance).

\section{The Reynolds' lubrication problem with load constraint}

A well known free boundary problem in elastohydrodynamic lubrication theory (see, for instance, [11], [7], [14]) consists of the determination of the nonnegative pressure $p=p\left(x_{1}, x_{2}\right)$ in a thin film of viscous fluid of constant density and pressuredependent viscosity $\mu=\mu(p)$ in an open bounded domain $x=\left(x_{1}, x_{2}\right) \in \Omega \subset \mathbf{R}^{2}$. In the presence of a cavitation region, where $p=0$, the flow region, which is a priori unknown, is determined by $p>0$ where the Reynolds' equation holds:

$$
Q(p) \equiv-\sum_{i=1}^{2} \frac{\partial}{\partial x_{i}}\left(\frac{h^{3}}{\mu} \frac{\partial p}{\partial x_{i}}\right)+\omega \frac{\partial h}{\partial x_{i}}=0 .
$$

Here $\omega=6 v>0$, if $v$ is the constant velocity, and $h=h[p]$ presents the positive film thickness, that may depend, in a nonlocal way, also on the pressure, which is assumed to satisfy the boundary condition

$$
p=0 \text { on } \partial \Omega
$$

In some cases, equation (3.1) must be modified in order to take also into account an additional load constraint (see [8])

$$
\int_{\Omega} p(x) d x=I
$$

for a prescribed constant $I>0$. We consider the change of variables 


$$
u=m(p)=\int_{0}^{p} \frac{d s}{\mu(s)}, \text { with inverse } p=q(u)
$$

where the viscosity $\mu$ is assumed to be a positive continuous function such that $q(m(p))=p=m(q(p))$ and

$$
q \text { is convex with } q(u)>0 \text { for } u>0 \text {. }
$$

We observe that, due to (3.4), we have the convexity of $q$ whenever $\mu$ is a nondecreasing function of the pressure.

We note that $\nabla u=\nabla p / \mu(p)$ and we assume from elastohydrodynamic lubrication theory a relation of the form

$$
H[u](x)=h[p](x)=h_{0}(x)+\int_{\Omega} \kappa(x, \xi) q(u(\xi)) d \xi
$$

where $h_{0}(x) \geq v>0$ is the given geometrical gap and the integral term takes into account the elastic deformation due to the fluid pressure with a nonnegative kernel $\kappa$.

Then, it is possible to reformulate the global problem in terms of a constrained unilateral problem in the subset

$$
K_{I}=\left\{v \in H_{0}^{1}(\Omega): v \geq 0 \text { in } \Omega, \int_{\Omega} q(v) d x=I\right\}
$$

for the transformed function $u=m(p)$, i.e.,

$$
u \in K_{I} \cap L^{\infty}(\Omega): \int_{\Omega}\left\{H^{3}[u] \nabla u+H[u] F\right\} \cdot \nabla(v-u) d x \geq 0, \forall \in K_{I}
$$

where $F=(\omega, 0)$. This nonlocal variational formulation is a natural generalization of the particular case considered in Section 4 of [14], where the viscosity was supposed to be a constant and, therefore, $q$ being linear, $K_{l}$ in (3.7) was a convex set.

We start by considering the simpler problem where the geometrical gap is prescribed by a function

$$
h \in L_{v}^{\infty}=\left\{g \in L^{\infty}(\Omega): g(x) \geq v>0 \text {, a.e. } x \in \Omega\right\},
$$

where $v>0$ is a given fixed constant, i.e., we replace (3.8) by

$$
u \in K_{I}: \int_{\Omega}\left(h^{3} \nabla u+h F\right) \cdot \nabla(v-u) d x \geq 0, \forall v \in K_{I}
$$

Introducing $K=\left\{v \in H_{0}^{\prime}(\Omega): v \geq 0\right.$ in $\left.\Omega\right\}$ and $\lambda \geq 0$, we shall consider first the auxiliary problem 


$$
\begin{aligned}
u_{\lambda} \in K: \int_{\Omega}\left(h^{3} \nabla u_{\lambda}+h F\right) & \cdot \nabla\left(v-u_{\lambda}\right) d x+\lambda \int_{\Omega} q(v) d x \\
& -\lambda \int_{\Omega} q\left(u_{\lambda}\right) d x \geq 0, \forall v \in K .
\end{aligned}
$$

As in [14], where the case $\lambda=0$ has been studied, we can prove the following

Proposition 3.1. For any $h \in L_{v}^{\infty}$ and any $\lambda \geq 0$, there exists a unique solution $u_{\text {, to }}$ $(3.10)_{\lambda}$, which is Hölder continuous in $\Omega$ and satisfies the estimates

$$
\begin{gathered}
\left\|\nabla u_{\lambda}\right\|_{L^{2}(\Omega)} \leq C_{\star} \equiv \omega v^{-2} \sqrt{|\Omega|}, \\
0 \leq u_{\lambda}(x) \leq C_{\sharp} \equiv 3 C_{2} C_{\star}, \forall x \in \Omega,
\end{gathered}
$$

where $F=(\omega, 0),|\Omega|=\operatorname{meas}(\Omega)$ and $C_{2}$ is the Sobolev constant of the inclusion $W^{1,1}(\Omega) \subset L^{2}(\Omega)$.

Proof. It follows from general results for the obstacle problem (see [12], for instance). In fact (3.11) is an easy consequence of letting $v=0$ in (3.10), and, remarking that $q\left(u_{\lambda}\right) \geq 0$, (3.12) follows exactly as in Proposition 2.2 of [14] by taking $v_{\lambda}=u_{\lambda}-\left(u_{\lambda}-\tau\right)^{+} \in K$ in (3.10) $)_{\lambda}$, with $\tau>C_{\#}$, since $q$ being nondecreasing, we have

$$
\lambda \int_{\Omega}\left[q\left(v_{\lambda}\right)-q\left(u_{\lambda}\right)\right] d x=\lambda \int_{\left\{u_{i}>\tau\right\}}\left[q(\tau)-q\left(u_{\lambda}\right)\right] d x \leq 0 .
$$

Remark 3.2. By (3.4) we have $p_{\lambda}=q\left(u_{\lambda}\right) \in K \cap C^{0, \alpha}(\bar{\Omega}), q^{\prime}\left(u_{\lambda}\right)=\mu\left(p_{\lambda}\right)$ and $\left\{u_{\lambda}>0\right\}=$ $\left\{p_{\lambda}>0\right\}$ are open subsets. Therefore, from $(3.10)_{\lambda}$, we can regard the associated pressure $p_{i}$ as the unique solution of the unilateral problem (recalling (3.1))

$$
\begin{gathered}
p_{\lambda} \geq 0, Q p_{\lambda}+\lambda \mu\left(p_{\lambda}\right) \geq 0 \text { in } \Omega \text { and } \\
Q p_{\lambda}+\lambda \mu\left(p_{\lambda}\right)=0 \text { in }\left\{p_{\lambda}>0\right\}
\end{gathered}
$$

with the boundary condition (3.2), for every $\lambda \geq 0$.

Remark 3.3. As observed in [7] and generalized in [14], the important case of the pressure-viscosity law of Barus

$$
\mu=\mu(p)=\mu_{0} e^{x p}, \mu_{0}>0, \alpha>0,
$$

can be included, provided $\alpha \mu_{0} C_{\#}<1$, where $C_{\#}$ is the constant of (3.12.) 


\section{CLASS OF ELLIPTIC PROBLEMS WITH NONCONVEX CONSTRAINTS 349}

In order to apply the abstract results of Section 1 we need to assure that the solution $u_{0}$ to $(3.10)_{0}$ with $\lambda=0$ is non trivial. In fact, if $u_{0}=0$ we obtain the necessary condition for the data

$$
\int_{\Omega} h F \cdot \nabla v d x \geq 0, \forall v \in K
$$

and we shall assume the additional assumption

$$
h \in M_{\delta}^{D}=\left\{q \in L^{\infty}(\Omega): \int_{D} q F \cdot \nabla \phi d x \leq-\delta \int_{D} \phi d x, \forall \phi \in \mathcal{D}(D), \phi \geq 0\right\}
$$

for some constant $\delta>0$ and some nonempty open subset $D \subset \Omega$. If $h$ is smooth, with $F=(\omega, 0),(3.15)$ is equivalent to the assumption

$$
\frac{\partial h}{\partial x_{1}} \geq \frac{\delta}{\omega}>0 \text { in } D \subset \Omega,
$$

a condition which is naturally satisfied, for instance, in the case of journal bearing where $h\left(x_{1}\right)=1+e \cdot \cos \left(x_{1}\right)$, with $0<e<1$ and $\left.\Omega=\right] 0,2 \pi[\times]-1,1[$.

Proposition 3.4. For any $h \in L_{v}^{\infty} \cap M_{\delta}^{D}$ let $u_{0}^{h}$ be the solution to (3.10). Then for any

$$
\left.I \in] 0, I_{0}^{h}\right], \text { with } I_{0}^{h}=\int_{\Omega} q\left(u_{0}^{h}\right) d x>0,
$$

there exists a unique $u=u^{h}$ solution to the pseudo-variational inequality (3.9). Moreover, it corresponds to a unique Lagrange multiplier $\lambda_{*}>0\left(\right.$ or $\lambda_{*}=0$ if $\left.u^{h}=u_{0}^{h}\right)$, with which $p_{\lambda_{*}}=q\left(u^{h}\right)$ is the unique solution of (3.13), (3.2).

Proof. It is easy to see that, by Proposition 1.5, we can take $I_{+}=I_{0}^{h}>0$, because $h \in M_{\delta}^{D}$ implies $u_{0}^{h} \not \equiv 0$. Observing that $I_{-}=0$ the Corollary 1.2 is immediately applicable to (3.9). By construction, $u^{h}=u \lambda_{\lambda}$ is the solution of (3.10), for a certain $\lambda=\lambda_{*} \geq 0$, and using the Remark 3.2 it remains to show the uniqueness of $\lambda_{*}>0$. Since $u_{\lambda_{*}}$ is unique, and $q$ is strictly increasing, the uniqueness of $\lambda_{*}$ is an immediately consequent of the equation (3.13)

$$
Q p_{\lambda_{+}}+\lambda_{*} \mu\left(p_{\lambda_{*}}\right)=-\nabla \cdot\left(h^{3} \nabla u^{h}+h F\right)+\lambda_{*} q^{\prime}\left(u^{h}\right)=0
$$

in $\left\{u^{h}>0\right\}=\left\{p_{\lambda}>0\right\}$.

Finally, using the a priori estimates (3.11) and (3.12), which are independent of $h \in L_{v}^{\infty}$, we can solve (3.8), by looking for a fixed point, for instance, in 


$$
\mathbf{C}=\left\{w \in H_{0}^{1}(\Omega): 0 \leq w \leq C_{\#},\|\nabla w\|_{L^{2}(\Omega)} \leq C_{*}\right\}
$$

and imposing additional assumptions on $h_{0}$ and on $\kappa$, so that, for $H(\mathbf{C}) \subset L_{v}^{\infty} \cap M_{\delta}^{D}$, for $H$ defined by (3.6). Let

$$
\begin{gathered}
h_{o} \in L_{v}^{\infty} \cap M_{\delta}^{D}, \kappa \geq 0 \text { in } \Omega, \frac{\partial \kappa}{\partial x_{1}} \geq 0 \text { in } D, \\
\kappa=\kappa(x, \xi) \in L^{\infty}\left(\Omega_{x}, L^{\prime}\left(\Omega_{\xi}\right)\right) \text { or } \kappa(x, \xi)=\kappa(x-\xi) \text { with } \kappa \in L_{l o c}^{1}\left(\mathbf{R}^{2}\right) .
\end{gathered}
$$

Theorem 3.5. Under the preceding assumptions, namely (3.4), (3.5), (3.6), (3.15), (3.18) and (3.19), there exists a positive number $I_{\star}$, depending only on the data $\Omega, D, \delta, v$, $\omega, \mu$, such that, for each

$$
\left.I \in \mathrm{]} 0, I_{\star}\right]
$$

there exists at least one solution to the problem (3.8).

Proof. Considering $H_{0}^{\prime}(\Omega)$ endowed with the weak topology, we can apply the Tychonov fixed point theorem in the compact convex set $\mathbf{C}$ given in (3.17) to the nonlinear continuous function $T: \mathrm{C} \rightarrow \mathrm{C}$, defined as follows: $T=P \circ H$, where $H$, given by (3.6), applies $C$ in a bounded subset of $L_{v}^{\infty} \cap M_{\delta}^{D}$, by the conditions (3.18), (3.19) and (3.5); $P$ applies to each $h \in L_{v}^{\infty} \cap M_{\delta}^{D}$, the unique solution $u_{h}$ of (3.9), which is in $C \cap K_{I}$ provided $I, \leq I_{0}^{h}$. The continuity of $T$ is an easy consequence of the continuous dependence of $u^{h}$ in $H_{0}^{1}(\Omega)$, when $h=H[w]$ varies in $L_{v}^{\infty} \cap M_{\delta}^{D}$ for the strong topology $L^{p}(\Omega), \forall p<\infty$, which happens when $w$ varies in C for the weak topology of $H_{0}^{1}(\Omega)$, by the compact embedding $H_{0}^{1}(\Omega) \subset L^{p}(\Omega), \forall p<\infty$, in two dimensions.

It remains to show that $I_{*}$ can be chosen positive and independent of $h=H[w]$, when $w$ varies in $\mathbf{C}$. Set

$$
I_{\star}=\inf _{w \in \mathbf{C}} I_{0}^{H[w]}=\inf _{w \in \mathbf{C}} \int_{\Omega} q\left(u_{0}^{H[w]}\right) d x
$$

where $u_{0}^{H[w]}$ is the unique solution to $(3.10)_{0}$ for $h=H[w]$. Since there exists $w_{*} \in \mathbf{C}$ solving (3.20) (taking a minimizing sequence $w_{n} \rightarrow w_{*}$ in $\mathrm{C}$, we have $u_{0}^{H\left[w_{n}\right]} \rightarrow u_{0}^{H\left[w_{*}\right]}$ in $H_{0}^{1}(\Omega)$, which implies $I_{0}^{H\left[w_{n}\right]} \rightarrow I_{0}^{H\left[w_{*}\right)}$ ) we conclude $I_{*}=I_{0}^{H\left[w_{0}\right]}>0$.

Consequently there exists a solution to $u=T u$, which solves the problem (3.8).

Remark 3.6. In fact, the mapping $T: \mathbf{C} \rightarrow \mathbf{C}$ is Lipschitz continuous, since if $u$ and $\hat{u}$ denote the solutions of (3.9) corresponding to $h=H[w]$ and $\hat{h}=H[\hat{w}]$, we have 


$$
\begin{aligned}
v^{3}\|\nabla(u-\hat{u})\|_{L^{2}(\Omega)}^{2} & \leq \int_{\Omega}\left\{\left(\hat{h}^{3}-h^{3}\right) \nabla u+(\hat{h}-h) F\right\} \cdot \nabla(u-\hat{u}) d x \\
& \leq\left(3 M^{2} C_{*}+\omega \sqrt{|\Omega|}\right)\|h-\hat{h}\|_{L^{\infty}(\Omega)}\|\nabla(u-\hat{u})\|_{L^{2}(\Omega)},
\end{aligned}
$$

where $\quad M=\sup _{w \in C}\|H[w]\|_{L^{\infty}(\Omega)}$. Set $q_{\sharp}=\max _{0 \leq v \leq c_{H}} q(v)>0$, assume for some $1<s<\infty, k_{s}=\|\kappa\|_{L^{\infty}\left(\Omega ; L^{s}(\Omega)\right)}<+\infty$ or $k_{s}=\|\kappa\|_{L^{s}(\Omega-\Omega)}<+\infty$ where $s^{\prime}=s /(s-1)$ and denote $C_{s}>0$ the constant of the Sobolev inclusion $H_{0}^{1}(\Omega) \subset L^{\mathrm{s}}(\Omega), \forall s<+\infty$. We have

$$
\|h-\hat{h}\|_{L^{\infty}(\Omega)} \leq q_{\| t} C_{s} k_{s}\|\nabla(w-\hat{w})\|_{L^{2}(\Omega)}
$$

and it follows that

$$
\|\nabla(u-\hat{u})\|_{L^{2}(\Omega)} \leq L\|\nabla(w-\hat{w})\|_{L^{2}(\Omega)},
$$

which, in particular, implies that $T$ is a strict contraction provided

$$
L=\left(3 M^{2} C_{*}+\omega \sqrt{|\Omega|}\right) q_{\#} C_{s} k_{s} v^{-3}<1 .
$$

Consequently, by the Banach fixed point theorem, (3.21) is a sufficient condition for the existence and uniqueness of the solution to (3.8). Under the strong restriction (3.21) and the above assumptions for $h_{0}$ and $\kappa$, the constraint $I$ may be chosen in ]0, $I_{0}$, where $I_{0}=\int_{\Omega} q\left(u_{0}\right) d x>0$ is defined through the solution $u_{0}$ of (3.10) for $h=H\left[u_{0}\right]$ and $\lambda=0$.

\section{A stationary problem with prescribed biological population}

In this section we consider an equilibrium problem for a spatially aggregating population model that is described by the convection-diffusion equation

$$
-\nabla \cdot(D(p) \nabla p-p V)=f,
$$

where the population density $p=p(x)>0$, for $x \in \Omega \subset \mathbf{R}^{N}, 1 \leq N \leq 3, \Omega$ being a given open bounded set. In (4.1) $f=f(x)$ is a given rate of births or deaths in the population and, in accordance with the biological model, the population flux $J=-D(p) \nabla p+p V$ includes both nonlinear diffusion and convection processes. We shall assume a diffusivity coefficient $D=D(p)$, such that it includes, in particular, the important case

$$
D(p)=d p^{m}, \text { where } d>0, m>0 \text { are constants, }
$$

and a nonlocal convection velocity of convolution type 


$$
V[p](x)=\int_{\Omega} Q(x-y) \sigma[p(y)] d y .
$$

where the vectorial kernel $Q$ takes into account the aggregative mechanism and $\sigma$ is a given real function. Our problem is inspired in a continuous version of Hamilton's model [6] following the approach of Mimura (see [10] for a study of the evolutionary one space dimension problem). Here we shall assume two additional features. Firstly, we do not know a priori where the population is located in $\Omega$, i.e., $\partial\{p>0\}$ is a free boundary and therefore we replace (4.1) by the associated zero-obstacle problem, as in the example of the preceding section. Secondly, we shall introduce an adjustable parameter, the total population $J>0$, that may be of practical importance, for instance in control problems or in the study of the asymptotic behaviour as in [10]:

$$
\int_{\Omega} p d x=J
$$

Finally, for simplicity, we shall assume a Dirichlet boundary condition

$$
p=0 \text { on } \partial \Omega \text {. }
$$

As in the previous section let us consider the change of variables

$$
u=m(p)=\int_{0}^{p} D(s) d s, \text { with the inverse } p=q(u)
$$

and assume the diffusivity coefficient $D(p)$ to be a positive continuous function on $p>0$ such that

$$
\begin{gathered}
q(u) \text { is concave with } q(u)>0 \text { and } q^{\prime}(u)>0 \text { for } u>0 \\
q(0)=0, q(u) \rightarrow+\infty \text { for } u \rightarrow+\infty, \\
|q(u)-q(v)| \leq C|u-v|^{\alpha}, \forall u, v \geq 0
\end{gathered}
$$

where $C>0$ and $1 / 2 \leq \alpha \leq 1$ are constants.

We note that if the diffusivity $D(p)$ is of the type (4.2), $q(u)=C_{\alpha} u^{\alpha}\left(C_{\alpha}>0, \alpha=\frac{1}{m+1}\right)$ satisfies the conditions (4.7)-(4.9).

Using $D(p) \nabla p=\nabla u$ and setting $b=\sigma \circ q$, the free boundary problem of biological population (4.1), (4.3)-(4.5) can be written in the following weak form

$$
u_{J} \in K_{J}: \int_{\Omega}\{(\nabla u-q(u) Q * b(u)) \cdot \nabla(v-u)+f(v-u)\} d x \geq 0, \forall v \in K_{J}
$$

where $K_{J}=\left\{v \in H_{0}^{1}(\Omega), v \geq 0\right.$ in $\left.\Omega, \int_{\Omega} q(v) d x=J\right\}$ is given as in (3.7), but now as the intersection of a convex set with the level set of a concave functional. 
Setting also $K=\left\{v \in H_{0}^{1}(\Omega), v \geq 0\right.$ in $\left.\Omega\right\}$ and considering now a negative parameter $\mu \leq 0$, for each

$$
\tau \in L_{+}^{2} \equiv\left\{v \in L^{2}(\Omega), v \geq 0 \text { a.e. in } \Omega\right\}
$$

we consider the auxiliary variational inequality for $w=u_{\tau, \mu}$ :

$$
\begin{array}{r}
w \in K: \int_{\Omega}\left\{\left[\nabla w-q(w) V_{\tau}\right] \cdot \nabla(v-w)+f(v-w)\right\} d x+\mu \int_{\Omega} q(v) d x \\
-\mu \int_{\Omega} q(w) d x \geq 0, \forall v \in K
\end{array}
$$

where $V_{\tau}=V_{\tau}(x)=V[q(\tau)]=Q * b(\tau)$ is given by (4.3) for $\tau \in L_{+}^{2}$.

In order to apply the results of the second example of Section 2 in the case (2.19) with $\gamma(x, u) \equiv f(x)$ and $B(x, u)=-q(u) V_{\imath}(x)$, we assume

$$
\begin{gathered}
f \in L^{2}(\Omega), Q \in\left[L^{1}\left(\mathbf{R}^{n}\right)\right]^{n}, \\
\exists \xi \in \mathbf{R}^{n} \backslash\{0\}: \xi \cdot Q(x) \leq 0(\text { or } \geq 0), \text { a.e. } x \in \mathbf{R}^{n}, \\
b=\sigma \circ q \in C^{0}\left(\left[0,+\infty[) \text { and } \exists \sigma_{*}<\infty: 0 \leq \sigma(t) \leq \sigma_{*}, \forall t \geq 0 .\right.\right.
\end{gathered}
$$

Proposition 4.1. Under the assumptions (4.7)-(4.9) with $\frac{1}{2} \leq \alpha<1$, (4.13)-(4.15), for each $\tau \in L_{+}^{2}$ and $\mu \leq 0$, there exists a unique $u_{\tau, \mu}$ solution of (4.12). Moreover $u_{\tau, \mu}$ is continuous and monotone in $\mu$, i.e.,

$$
\text { if } \mu_{1}<\mu_{2} \leq 0 \text { then } u_{\tau, \mu_{1}} \geq u_{\tau, \mu_{2}} \text { a.e. in } \Omega
$$

and, for each $\tau \in L_{+}^{2}$, satisfies the following estimate

$$
\frac{1}{2} \int_{\Omega}\left|\nabla u_{\tau, \mu}\right|^{2} d x \leq C_{0}-\mu \int_{\Omega} q\left(u_{t, \mu}\right) d x
$$

where the constant $C_{0}>0$ depends only on $\alpha, f, \sigma_{*}, \Omega$ and $\|Q\|_{L^{1}}$

Proof. The estimate (4.17) is a simple consequence of (4.12) with $v=0$, since by Poincaré and Young inequalities we have 


$$
\begin{aligned}
\int_{\Omega} f w d x-\int_{\Omega} q(w) V_{\tau} \cdot \nabla w d x & \leq\|f\|_{L^{2}}\|w\|_{L^{2}}+\left\|V_{\tau}\right\|_{L^{\infty}}\|q(w)\|_{L^{2}}\|\nabla w\|_{L^{2}} \\
& \leq C\|f\|_{L^{2}}\|\nabla w\|_{L^{2}}+C_{\alpha} \sigma_{*}\|Q\|_{L^{1}}\|w\|_{L^{2}}^{\alpha}\|\nabla w\|_{L^{2}} \\
& \leq \frac{1}{2}\|\nabla w\|_{L^{2}}^{2}+C_{0}\left(C\|f\|_{L^{2}}, C_{x} \sigma_{*}\|Q\|_{L^{1}}, \alpha\right)
\end{aligned}
$$

where we have used the growth condition $\alpha<1$. Consequently the elliptic operator associated with (4.12) is of the form (2.2) and is coercive in the sense of (1.5). It is also clear that the assumptions (4.7), (4.9) with $\alpha \geq 1 / 2,(4.14)$ and (4.15) allow us to apply the Proposition 2.2. Since $\mu q=\lambda \psi$ is a convex and decreasing function, the corresponding comparison principle, as in Proposition 2.3, yields now the conclusion (4.16).

In order to apply the Theorem 1.4, it is sufficient to observe that the Assumption I is satisfied, since the operator is coercive and now $\operatorname{Dom} \Psi=K$, being $\Psi(v)=\int_{\Omega} q(v) d x$ continuous for the weak topology of $H_{0}^{1}(\Omega)$, by the continuity of $q$ and the compact embedding $H_{0}^{1}(\Omega) \subset L^{2}(\Omega)$.

The assumption (1.10) holds, since $J_{+}=+\infty$ and

$$
0 \leq J_{-} \equiv \inf _{\mu \leq 0} \int_{\Omega} q\left(u_{\tau, \mu}\right) d x=\int_{\Omega} q\left(u_{\tau, 0}\right) d x \equiv J_{\tau}<+\infty,
$$

where $u_{\tau, 0}$ denotes the unique solution to (4.12) with $\mu=0$ and $\tau \in L_{+}^{2}$. We observe that $J_{\tau}=0$ if $f \leq 0$, since then $u_{\tau, 0} \equiv 0$ solves $(4.12)_{0}$ for any $\tau \in L_{+}^{2}$. On the other hand, the estimate (4.17) with $\mu=0$, yields the conclusion

$$
0 \leq \sup _{\tau \in L_{+}^{2}} J_{\tau} \equiv J_{x}<+\infty
$$

where $J_{\alpha}$ is a constant depending only of $\alpha, \Omega$ and the constant $C_{0}$ of (4.17).

Proposition 4.2. Let the assumptions of Proposition 4.1 hold. Then for any $\tau \in L_{+}^{2}$ and for each

$$
J \in\left[J_{\tau},+\infty[\right.
$$

where $J_{\tau}$ is defined by (4.19), there exists a unique pair $w=u_{t, \mu} \in K$ and $\mu=\mu_{\tau} \in$ $\left[-M_{J}, 0\right]$ solving $(4.12)$ and such that

$$
\int_{\Omega} q\left(u_{t, \mu}\right) d x=J
$$

Moreover, for each $J \geq J_{x}, J_{\alpha}$ given by (4.20), the constant $M_{J}$, such that, $0 \geq \mu_{\tau} \geq-M_{J}$ 
is independent of $\tau \in L_{+}^{2}$ and the application $L_{+}^{2} \ni \tau \mapsto\left\{u_{\tau, \mu}, \mu_{\tau}\right\} \in K_{J} \times\left[-M_{J}, 0\right]$ is continuous.

Proof. The existence of $u_{\tau, \mu}$ and $\mu_{\tau}$, by the assumptions, is a corollary of the Theorem 1.4. Consequently it yields the existence of a solution $u_{\tau}$ to the problem

$$
u=u_{\tau} \in K_{J}: \int_{\Omega}\{(\nabla u-q(u) Q * b(\tau)) \cdot \nabla(v-u)+f(v-u)\} d x \geq 0, \forall v \in K_{J}
$$

However, we are unable to guarantee the uniqueness for (4.23) but the pair $\left\{u_{\tau, \mu}, \mu_{\tau}\right\}$ is the unique solution of (4.12), such that $u_{t, \mu_{4}} \in K_{J}$.

Indeed, if we have two different solutions $\left\{u_{1}, \mu_{1}\right\}$ and $\left\{u_{2}, \mu_{2}\right\}$ satisfying (4.22) and such that, for instance, $\mu_{1}<\mu_{2}$, by comparison, we have $u_{1}>u_{2}$ a.e. in $\Omega$, and by the strict monotonicity of $q$ it follows that $u_{1}=u_{2}$ a.e. in $\Omega$. But if there exists an open subset $\omega \subset \Omega$ where $u_{1}(x)=u_{2}(x)>0$ for a.e. $x \in \omega$, we have

$$
-\nabla \cdot\left[\nabla u_{1}-q\left(u_{1}\right) V_{\mathrm{r}}\right]+\mu_{1} q^{\prime}\left(u_{1}\right)=f \text { a.e. in } \omega,
$$

and from the analogous equation for $\left\{u_{2}, \mu_{2}\right\}$, by subtraction, we obtain

$$
\mu_{1} q^{\prime}\left(u_{1}\right)=\mu_{2} q^{\prime}\left(u_{2}\right) \text { a.e. in } \omega \text {. }
$$

Then, using the assumption (4.7) we should have also $\mu_{1}=\mu_{2}$, which is a contradiction.

To see that $\mu_{2}$ varies in a bounded interval when $\tau$ varies in $L_{+}^{2}$, we write (4.12) for $\mu<0$ in the form

$$
\begin{aligned}
\int_{\Omega} q(w) & \geq \int_{\Omega} q(v)-\frac{1}{\mu}\left\{\int_{\Omega}|\nabla w|^{2}-\int_{\Omega}\left[\nabla w \cdot \nabla v+q(w) V_{\tau} \cdot \nabla(v-w)+f(v-w)\right]\right\} \\
& \geq \int_{\Omega} q(v)-\frac{1}{\mu}\left\{\frac{1}{2} \int_{\Omega}|\nabla w|^{2}-C^{\prime} \int_{\Omega}|\nabla v|^{2}-C^{\prime \prime} \int_{\Omega} f^{2}\right\} \\
& \geq \int_{\Omega} q(v)+\frac{1}{\mu}\left\{C^{\prime} \int_{\Omega}|\nabla v|^{2}+C^{\prime \prime} \int_{\Omega} f^{2}\right\}, \forall v \in H_{0}^{1}(\Omega), v \geq 0,
\end{aligned}
$$

where, as in the estimate (4.18), $C^{\prime}$ and $C^{\prime \prime}$ are positive constants independent of $\mu$ and $\tau$. Taking $v_{\mu}=|\mu|^{1 / 4} v_{*}$ in (4.24), for some $v_{*} \in H_{0}^{1}(\Omega)$ such that $v_{*} \geq 1$ a.e. in some open $\omega \subset \Omega, \omega \neq \emptyset$, by the assumption (4.7) we find, for all $\mu<-1$ :

$$
\varphi_{\tau}(\mu) \equiv \int_{\Omega} q\left(w_{\tau, \mu}\right) \geq \int_{\omega} q\left(|\mu|^{1 / 4}\right)-C_{*}|\mu|^{-1 / 2}
$$

where $C_{*}>0$ is independent of $\mu$ and $\tau$. Using the assumption (4.8) we see that when we take $|\mu|$ sufficiently large in order to obtain the root of the equation $\varphi_{\imath}\left(\mu_{\tau}\right)=J$, this 
can be done uniformly in $\tau \in L_{+}^{2}$ and therefore there exists a constant $M_{J}<+\infty$, such that $\mu_{\tau} \geq-M_{\jmath}, \forall \tau \in L_{+}^{2}$.

The continuous dependence with respect to $\tau$ is an easy consequence of the uniqueness of (4.12) and of the continuity of $v \mapsto \int_{\Omega} q(v) d x$.

We can now state the main result of this section.

Theorem 4.1. Under the preceding assumptions, namely, (4.7)-(4.9) with $\frac{1}{2} \leq \alpha<1$, (4.13)-(4.15) and with $J_{\alpha}$ defined by (4.20), for each

$$
J \in\left[J_{\alpha},+\infty[\right.
$$

there exists at least a solution $u_{J}$ to the pseudo-variational inequality (4.10).

Proof. Set $C_{R}=\left\{\tau \in L_{+}^{2}:\|\tau\|_{L^{2}} \leq R\right\}$ and define the application $T: \tau \mapsto$ $u_{\tau, \mu_{\tau}}=T(\tau)$, where $u_{\tau, \mu_{\tau}}$ is the unique solution of (4.12) with $\mu=\mu_{\tau}$ such that (4.22) is satisfied. This operator is continuous and, from (4.17), $u_{\tau, \mu_{t}} \in K_{J}$ satisfies the estimate

$$
\frac{1}{2} \int_{\Omega}\left|\nabla u_{\tau, \mu_{2}}\right|^{2} \leq C_{0}-\mu_{\tau} J \leq C_{0}+M_{J} J
$$

which is independent of $\tau$, by the Proposition 4.2. Consequently, for large $R$, $T\left(C_{R}\right) \subset C_{R}$ and, by the compactness of the injection $H_{0}^{1}(\Omega) \subset L^{2}(\Omega), T$ is also compact.

Hence, by the Schauder fixed point theorem, there is a $u_{J}$ such that $u_{J}=T u_{J}$, which is a solution to $(4.10)$.

Remark 4.1. In particular for the case (4.2), we have given conditions for the existence of a solution of a free boundary problem (see Remark 2.4) consisting of finding a nonnegative function $p$ and a subset $\{p>0\} \subset \Omega$, where the population is located in equilibrium and satisfies the equation

$$
-\nabla \cdot\left(d p^{m} \nabla p-p V[p]\right)+\mu p^{-m}=f \text { in }\{p>0\}
$$

under the side constraint (4.4), where $\mu \leq 0$ is a generalized Lagrange multiplier and $0<m \leq 1$.

Remark 4.2. We have considered only the case $\frac{1}{2} \leq \alpha<1$, i.e., $0<m \leq 1$. However it is also possible to consider $\alpha=1$ in Theorem 4.1 provided $\sigma_{*}\|Q\|_{L^{\prime}}$ is sufficiently small, such that (4.18) still holds. 


\section{REFERENCES}

1. H. Berestyki, J. M. Coron and I. E. Ekeland (Editors), Variational Methods (Birkhäuser, Boston, 1990).

2. H. BrÉzIS, Équations et inequations nonlinéaires dans les espaces vectoriels en dualité, Ann. Inst. Fourier (Grenoble) 18 (1968), 115-175.

3. M. Chipot and G. Michaile, Uniqueness results and monotonicity properties for strongly nonlinear elliptic variational inequalities, Ann. Scuola Norm. Sup. Pisa 16 (1989), 137-166.

4. G. Duvaut and J. L. Lions, Inequalities in Mechanics and Physics (Springer-Verlag, Berlin, 1976).

5. D. Gilbarg and N. S. Trudinger, Elliptic Partial Differential Equations of Second Order, $2^{\text {nd }}$ ed. (Springer-Verlag, Berlin, 1983).

6. W. D. Hamilton, Geometry for the selfish herd, J. Theoret. Biol. 31 (1971), 295-311.

7. B. Hu, A quasi-variational inequality arising in elastohydrodynamics, SIAM J. Math. Anal. 21 (1990), 18-36.

8. M. M. Kostreva, Pressure spikes and stability considerations in elastrohydrodynamic lubrication models, Trans. ASME - J. Tribology 106 (1984), 386-391.

9. J. L. Lions, Quelques Méthodes de Résolution des Problèmes aux Limites Non Linéaires (Dunod, Paris, 1969).

10. M. Mimura, Some convection-diffusion equations arising in population dynamics, Contemp. Math. 17 (1983), 343-351.

11. J. T. ODEN and S. R. WU, Existence of solutions to the Reynolds' equation of elastohydrodynamic lubrication, Internat. J. Engng. Sci. 23 (1985), 207-215.

12. J. F. Rodrigues, Obstacle Problems in Mathematical Physics (North-Holland, 1987).

13. J. F. Rodrigues, Some remarks in the quasi-linear noncoercive elliptic obstacle problem, Pitman Res. Notes Math. Ser. 208 (1989), 319-332.

14. J. F. RoDrigues, Remarks on the Reynolds problem of elastohydrodynamic problem, European J. Appl. Math. 4 (1993), 83-96.

15. E. ZeIdLeR, Nonlinear Functional Analysis and its Applications, Vol III - Variational Methods and Optimization (Springer-Verlag, New York, 1985).

Departamento de Matemática

UNIVERSIDADE INDEPENDENTE

Av. Marechal Gomes da CoSta, 9

P-1800 LisBoa

PORTUGAL
Centro de Matemática e Apliçacões Fundamentais UNIVERSIDADE DE LISBOA

Av. Prof. Gama Pinto, 2

P-1699 LisBoa CODEX

Portugal 